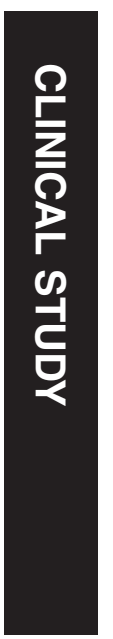

${ }^{1}$ Eye Center, Zhejiang Provincial People's Hospital, Hangzhou, China

${ }^{2}$ Wilmer Eye Institute, Johns Hopkins Hospital, Baltimore, MD, USA

${ }^{3}$ Glaucoma Consultants, Baltimore, MD, USA

${ }^{4}$ Vistar Eye Center, Roanoke, VA, USA

${ }^{5}$ Walter Reed National Military Medical Center, Bethesda, MD, USA

${ }^{6}$ Department of Biostatistics, Johns Hopkins Bloomberg School of Public Health, Baltimore, MD, USA

Correspondence: Q Pan, Eye Center, Zhejiang Provincial People's Hospital, 158 Shangtang Road, Hangzhou 310014, China

Tel: +86 57185239988 ; Fax: +86 57185893015 . E-mail: panqing@hotmail. com

Received: 10 March 2016 Accepted in revised form: 22 September 2016

Published online:

11 November 2016

\section{Clinical outcomes of gamma-irradiated sterile cornea in aqueous drainage device surgery: a multicenter retrospective study}

Q Pan ${ }^{1,2}$, HD Jampel ${ }^{2}$, P Ramulu², GF Schwartz ${ }^{3}$, F Cotter ${ }^{4}$, D Cute ${ }^{5}$, YJ Daoud ${ }^{2}$, P Murakami ${ }^{6}$ and WJ Stark ${ }^{2}$

\begin{abstract}
Purpose The purpose of the study was to evaluate the safety and efficacy of gammairradiated sterile cornea (GISC) for covering the tube in aqueous drainage device (ADD) surgery in a retrospective, multicenter case series.
\end{abstract}

Patients and methods Participants included 297 patients (321 procedures) who had undergone ADD surgery for the first time using GISC patch at three clinic centers in the United States between April 2009 and July 2012. The medical records of those consecutive patients were reviewed. Preoperative, intraoperative, and postoperative parameters about GISC were collected and analyzed. The main outcome measures were patch graft failure (PGF) and postoperative complications related to GISC.

Results Three hundred and nineteen eyes in 295 patients were included in the current analysis. Ten out of the 319 eyes experienced PGF with a mean follow-up of $15.4 \pm 9.8$ (SD) months. The overall cumulative PGF proportion from KaplanMeier analysis was $2.6 \%$ (95\% CI: $0.6-4.7 \%$ ) at 18 months. We detected two cases of presumed endophthalmitis related to PGF.

Conclusions GISC appears to have a reasonable success rate for preventing tube exposure related to PGF over an 18-month period. This success rate, in combination with other features of GISC (transparency and storage at room temperature), makes it a viable choice for patch graft material during ADD.

Eye (2017) 31, 430-436; doi:10.1038/eye.2016.230; published online 11 November 2016

\section{Introduction}

Aqueous drainage device (ADD) implantation is an effective surgery for glaucoma,,$^{1,2}$ but tube erosion through the overlying patch and conjunctiva is a potentially serious complication and a risk factor for the development of endophthalmitis. Therefore, a variety of materials, including sclera, ${ }^{3}$ dura mater, ${ }^{4}$ fresh cornea, ${ }^{5,6}$ fascia lata, ${ }^{7}$ pericardium, ${ }^{8}$ and glycerol-preserved cornea, ${ }^{9}$ have been used to cover the portion of the tube lying underneath the conjunctiva to prevent erosion. Each of these materials has at least some disadvantages, including potential transmission of infectious disease, graft melting, opaqueness, and difficulty in surgical manipulation. $6,7,10,11$

The ideal material would be immunologically safe, biocompatible, stable, and cosmetically acceptable. Gamma-irradiated sterile cornea (GISC) fulfills all these criteria owing to its unique features. GISC is cryogenically and chemically treated, stabilized in media, and then gamma-irradiated for total sterility. This processing results in a reduced risk of infection transmission and decreased alloantigen load by devitalizing corneal cells. ${ }^{12}$ Additional advantages of GISC include shelf-stability at room temperature for up to 2 years and transparency with high tensile strength. GISC is currently provided in various shapes and sizes with full- or partial-thickness stroma for ophthalmic surgery use. GISC has been successfully demonstrated to have good biocompatibility when used for glaucoma patch graft, corneal lamellar keratoplasty, and as a biological skirt for keratoprosthesis. ${ }^{13-15}$

The use of GISC as coverage patch in primary or secondary ADD surgery with short follow-up 
of $4.8 \pm 3.5$ months has been recently reported; ${ }^{16}$ however, GISC is a relatively new material and the long-term clinical results in coverage of ADD are limited. To better evaluate the safety and effectiveness of the GISC as patch graft in ADD surgery, we performed a multicenter retrospective study, paying special attention to the function of GISC as tube coverage to prevent erosion and other GISC-related complications over a mean follow-up of 15.4 months.

\section{Materials and methods}

This is a retrospective, multicenter review of patients who underwent ADD implantation surgery for the first time, using GISC as a patch by experienced surgeons, at 3 tertiary referral centers in the United States between April 2009 and July 2012. The tertiary referral centers include the Wilmer Eye Institute/Johns Hopkins Hospital (Baltimore, MD), Glaucoma Consultants (Towson, MD), and Vistar Eye Center (Roanoke, VA). The protocol for this study underwent approval by Institutional Review Boards (IRBs) at Johns Hopkins Medical Institution and the Western Institutional Review Board's IRB Affairs Department. The inclusion criteria were: (1) patients undergoing first-time ADD surgery with one GISC patch graft, (2) a minimum follow-up of 4 months and (3) eyes without a keratoprosthesis.

\section{Evaluation criteria}

We defined patch graft failure (PGF) as tube erosion through the patch graft and overlying conjunctiva. Because the sole function of GISC as tube coverage is to prevent erosion, the term 'patch graft failure' ${ }^{\prime 17}$ succinctly characterizes this complication and is used in the current study. Time to PGF or administrative censoring (last follow-up) was defined as the interval between tube shunt surgery and the date of graft exchange, tube explantation, or last follow-up.

\section{Main outcome measurements}

The primary outcome in the current study was PGF. PGF and other outcomes related to GISC were assessed by reviewing preoperative, intraoperative, and postoperative medical records.

\section{Graft materials}

The GISC graft (VisionGraft) is a cornea allograft from a medically qualified donor that Tissue Banks International (Baltimore, MD, USA) has certified to be in compliance with Eye Bank Association of America standards. The corneal grafts are first stored frozen. The tissues are then precut in the shape of a split thickness half-moon, according to the surgeon's needs for ADD surgery (Figure 1a), and then sent for bioburden testing. Thereafter, the tissues are sterilized with a validated gamma-irradiation process. The sterility is further verified by microbiology testing (DEW, BA, written communication, 18 February 2011). They are stored in albumin, with a shelf life of up to 2 years at room temperature.

\section{Surgical procedure}

ADD implants (Ahmed, Baerveldt, or Molteno) were placed in the superotemporal or inferotemporal quadrants using a fornix-based incision without intraoperative mitomycin C. GISC grafts were sutured in place with 10-0/9-0 nylon or 6-0 to 8-0 Vicryl sutures (Figure 1b).

\section{Statistical analysis}

Univariate Cox-proportional hazard models, with robust SEs based on a grouped jackknife to account for clustering (the fact that in some cases both eyes of the same patient were analyzed), were used to evaluate potential risk factors associated with a higher rate of PGF. KaplanMeier survival analysis, with SEs obtained using the formula of Ying and $\mathrm{Wei}^{18}$ to account for clustering, was performed to estimate the proportion of eyes without PGF at various time points after surgery. A $P$-value of $<0.05$ was considered statistically significant, and all tests were two-sided and based on complete-case analysis. All analyses were performed using the R statistical software v3.0.2 (R Foundation, Auckland, New Zealand).

\section{Results}

Between April 2009 and July 2012, there were 321 consecutive ADD surgeries with GISC as the patch graft in 297 patients, which were operated on by 12 experienced surgeons at the 3 mentioned hospitals. Of these, 2 eyes in 2 patients were excluded, due to $<4$ months follow-up (no erosion occurred during the F/U). Therefore, 319 ADD implantation surgeries for the first time, with GISC patch, performed in 295 patients, were entered into the study sample.

Patients' demographic characteristics are summarized in Table 1. The mean age of the patients was $63.6 \pm 19.1$ (SD) years (range: 0.9-98.8years) at the time of surgery. In all, 51.2\% (151 patients) were Caucasian, 37.3\% (110 patients) African descent, and $11.5 \%$ (34 patients) other (including Hispanic, Asian, and unknown). The overall indications for surgery were: primary open-angle glaucoma (156, 48.9\%), neovascular glaucoma (45, 

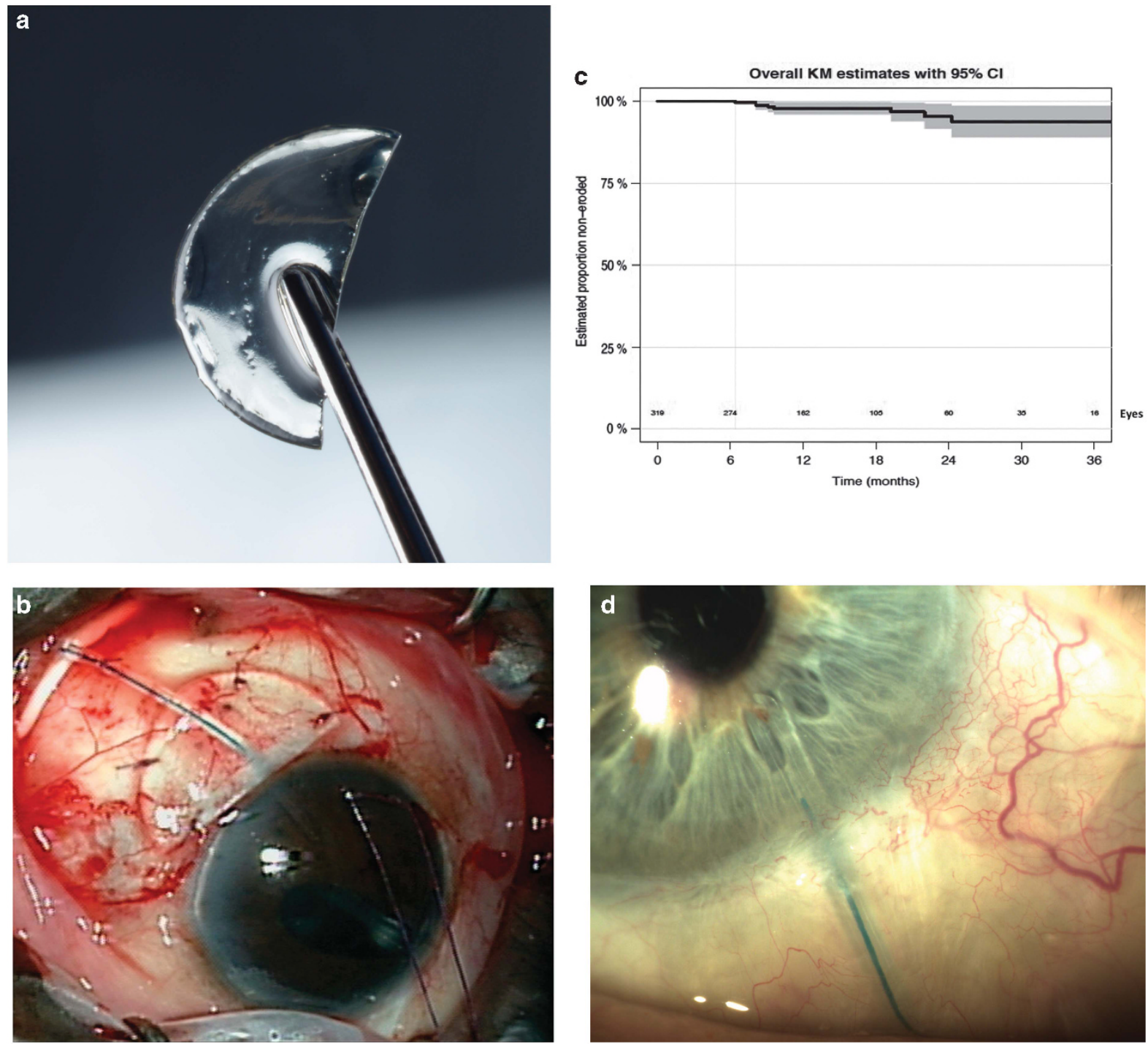

Figure 1 (a) GISC is precut for ADD surgery: a piece of split half-moon GISC tissue. (b) The GISC graft was sutured to sclera to cover the tube. (c) Kaplan-Meier curve demonstrating GISC graft survival as a function of time. (d) Slit-lamp photographs illustrating the appearance of GISC patch at 9 months after ADD surgery. The tube underneath is observable through the transparent GISC patch during the follow-up.

$14.1 \%)$, uveitic glaucoma $(32,10.0 \%)$, steroid response glaucoma (26, 8.2\%), pseudoexfoliation glaucoma $(15,4.7 \%)$, primary congenital/juvenile glaucoma $(11,3.4 \%)$, pigmentary glaucoma $(7,2.2 \%)$, traumatic glaucoma $(7,2.2 \%)$, primary angle-closure glaucoma $(6,1.9 \%)$, and other secondary glaucoma $(14,4.4 \%$;

Table 1).

Ten out of the 319 eyes experienced PGF over a mean follow-up of 15.4 \pm 9.8 (SD) months (range: 4.1-43.9 months). The median time to PGF for these 10 eyes was 13.8 \pm 7.4 (SD) months (range: 6.4-24.2 months) after surgery. Figure 1c shows that the cumulative proportions of PGF, as calculated by the Kaplan-Meier curve, were $0 \%$ at 6 months and 2.6\% (95\% CI: $0.55-4.71 \%)$ at both 12 and 18 months.

Table 2 presents the characteristics and management of 10 cases of PGF. There were two cases of presumed endophthalmitis following PGF-both were culture negative, and both resolved after receiving intravitrous injections of antibiotic and steroid. ADD shunts were explanted in five cases of PGF owing to iris plugging tube or presumed endophthalmitis. Two grafts among the 10 cases of PGF were described to be 'nonexistent' during the revision surgery.

There were 7 cases of tube/plate exposure with intact GISC graft, including 5 cases (1.6\%) of plate exposure, 1 
Table 1 Patients demographics

\begin{tabular}{lc}
\hline Characteristic & No. \\
\hline Patients & 295 \\
Eyes & 319 \\
Right/left & $162 / 157$ \\
Gender: male/female & $132 / 163$ \\
& \\
Race & \\
Caucasian/African descent/others & $151 / 110 / 34$ \\
& \\
Mean age \pm SD (years) at surgery & $63.6 \pm 19.1$ \\
& (range: $0.9-98.8$ years) \\
Type of glaucoma diagnosis & \\
Primary glaucoma & 162 \\
Primary open-angle glaucoma & 156 \\
Primary angle-closure glaucoma & 6 \\
Secondary glaucoma & 157 \\
Neovascular glaucoma & 45 \\
Pseudoexfoliation glaucoma & 15 \\
Pigmentary glaucoma & 7 \\
Traumatic glaucoma & 7 \\
Uveitic glaucoma & 32 \\
Steroid responder glaucoma & 26 \\
Congenital glaucoma/juvenile & 11 \\
glaucoma & \\
Other secondary glaucoma & 14 \\
\hline
\end{tabular}

case of tube exposure with graft retraction $(0.3 \%)$, and 1 case of tube-eroded posterior to GISC (0.3\%). As these seven cases of tube/plate exposure were covered with intact GISC graft, they were not counted as PGF.

Three eyes in this cohort received laser suture lysis through transparent GISC between 5 and 7 weeks after ADD surgery.

The risk factors considered in the univariate analysis for PGF included age ( $\leq 18$ years $v s \geq 18$ years of age), glaucoma diagnosis, number of prior glaucoma medications, thyroid, systemic autoimmune disease (rheumatoid arthritis, etc.), diabetes, other systemic morbidities (hypertension, etc.), smoking, family history of glaucoma, contact lens wearer, ocular surface disease (including pyterygium, dry eye, conjunctivitis, etc.), other ocular diseases (including cataract, uveitis, retinal vasculitis, etc.), type of suture used for graft or shunt (nylon vs Vicryl), shunt type (Ahmed, Baerveldt, or Molteno), plate location (inferior/superior), intraoperative complication (hyphema, shallow anterior chamber, etc.), trabeculectomy/exPress shunt/bleb revisions, other prior glaucoma surgeries, other prior intraocular surgeries (including cataract surgery, penetrating keratoplasty, eyelid surgery, retinal detachment repair with scleral buckle, vitrectomy with/ without silicone oil, etc.), or concomitant surgeries (including cataract extraction and insertion of an intraocular lens, trabeculectomy, pars planavitrectomy, intravitrous silicone oil removal, intravitreous injection, bleb needling/revision, etc.). However, none of the associations between these risk factors and PGF was found to be statistically significant based on univariate Cox-proportional hazards models in the current sample (all $P>0.05$, data not shown).

\section{Discussion}

The current retrospective study demonstrates the efficacy and safety of using GISC as patch graft material in a large series of consecutive ADD implantation surgery for the first time. The overall cumulative proportions of PGF, as calculated from the Kaplan-Meier curve (Figure 1c), were $0 \%$ at 6 months and $2.6 \%$ at both 12 and 18 months, which fall within the range of $0-7 \%$ in the literature on PGF in various patch materials (such as pericardium, sclera, dura, etc.) with follow-up averaging 10-16 months. ${ }^{1,4,6,7,19-24}$

GISC generally remained intact over the tube and appeared well tolerated during this study. There were 2 grafts among the 319 cases $(0.6 \%)$ documented nonexistent during the revision surgery for tube erosion, though none of GISC were noted as thinning during the follow-up period. Smith et $a l^{24}$ and Raviv et al ${ }^{19}$ reported that patch thinning occurred frequently in the pericardium group (26.1\%, follow-up of 33 months), the dura group (22.2\%, follow-up of 47 months), and the sclera graft (26.1\%, follow-up of 66 months). The covering materials were thought prone to silently melt if they fail to integrate with the host, which is possibly owing to a slow immune-related process. ${ }^{24}$ This may eventually lead to loss of tectonic strength and increased risk of tube erosion, even in the absence of predisposing factors. It is worthy of noting that surgeons usually judge sufficient patch thinning by observing whether the sutures securing the tube to sclera could be visible through those opaque material grafts. In this context, it would be difficult for ophthalmologists to notice the thinning in a transparent GISC. Also, it is impossible for surgeons to observe GISC grafts in those eyes with particularly thick conjunctiva and Tenon's capsule, therefore, the true incidence of GISC thinning/melting might well be higher than noted $(0.6 \%)$ in this cohort. A prospective study quantifying the thickness of GISC with anterior segment optical coherence tomography may help determine GISC thinning/melting after ADD surgery.

It has been reported that clear patch tissue facilitates laser suture lysis in eyes with shunt implants, without using releasable sutures or instrumentation. ${ }^{5}$ GISC remains transparent, owing to the crosslinking process, when irradiated with gamma rays. ${ }^{25,26}$ Three cases in the current cohort underwent laser suture lysis after ADD 
Table 2 Summary of 10 PGF cases

\begin{tabular}{|c|c|c|c|c|c|}
\hline Case & Diagnosis & Surgeries prior to ADD (Months) & $\begin{array}{l}\text { Time to } \\
\text { PGF }\end{array}$ & Management & Endophthalmitis \\
\hline 1 & POAG & Phaco/IOL & 24.3 & $\begin{array}{l}\text { Explanation +replant } \\
\text { Baerveldt+new } \\
\text { GISC+conjunctiva autograft }\end{array}$ & $\mathrm{N}$ \\
\hline 2 & UV & Glaucoma none & 19.2 & Explanation & $\mathrm{N}$ \\
\hline 3 & PACG & Trabeculectomy+Phaco/IOL & 8.1 & Explanation & $Y^{a}$ \\
\hline 4 & Steroid glaucoma & Trabeculectomy+Phaco/IOL+new GISC & 6.4 & $\begin{array}{l}\text { Explanation+replant } \\
\text { Baerveldt }\end{array}$ & $\mathrm{N}$ \\
\hline 5 & POAG & Trabeculectomy+MMC & 9.1 & Pericardial allograft & $\mathrm{N}$ \\
\hline 6 & POAG & Trabeculectomy+repair & 8.1 & New GISC & $\mathrm{N}$ \\
\hline 7 & POAG & Trabeculectomy+repair+Phaco/IOL & 9.6 & Sclera graft & $\mathrm{N}$ \\
\hline 8 & Pseudoexfoliation & Trabeculectomy+MMC+Phaco/IOL & 22.0 & Explanation & $\mathrm{N}$ \\
\hline 9 & NVG & Intravitreal injection of Avastin & 8.0 & Explanation & $\mathrm{N}$ \\
\hline 10 & PACG & $\begin{array}{l}\text { Multiple retinal surgeries (including: vitrectomy } \\
\text { +silicone oil, etc.) }\end{array}$ & 23.3 & New GISC & $\mathrm{Y}^{\mathrm{a}}$ \\
\hline
\end{tabular}

${ }^{a}$ The microbial culture report showed that no microbial grew from either the vitreous specimen or the tube itself.

Table 3 PGF rate of various graft patches

\begin{tabular}{|c|c|c|c|c|c|c|c|c|}
\hline Study & Graft & Patients & Eyes & $F / U$ & Tube & PGF rate & PGF time & Age \\
\hline Pan & GISC & 295 & 319 & $15.4 \pm 9.8$ months & Various & $2.6 \%{ }^{a}$ & $13.8 \pm 7.4(6.4-24.2)$ months & $63.6 \pm 19.1$ years \\
\hline \multirow[t]{2}{*}{ Zalta $^{17}$} & Dura & 695 & 695 & $9.5 \pm 3.7$ years & Ahmed & $6.3 \%$ & $5.8 \pm 3.8(0.13-13.6)$ years & $62.3 \pm 18.1$ years \\
\hline & Sclera & 1121 & 1121 & $4.2 \pm 2.1$ years & & $5.8 \%$ & $2.7 \pm 1.9(0.07-8.4)$ years & $62.3 \pm 18.1$ years \\
\hline \multirow[t]{2}{*}{ Wigton et $a l^{9}$} & Cornea $^{\mathrm{b}}$ & 161 & 161 & $440(160-715)$ days & Various & $1.9 \%$ & 440 (158-706) days & $65.6 \pm 14.7$ years \\
\hline & Pericardium & 101 & 101 & 331 (69-567) days & & $8.9 \%$ & 252 (57-549) days & $65.7 \pm 15.3$ years \\
\hline \multirow[t]{3}{*}{ Smith et $a l^{24}$} & Sclera & 23 & 23 & $66 \pm 13$ months & Various & $4.3 \%$ & 15 months & 67.9 years \\
\hline & Dura & 18 & 18 & $47 \pm 4$ months & & $5.6 \%$ & 6 months & 69.5 years \\
\hline & Pericardium & 23 & 23 & $33 \pm 6$ months & & 0 & NA & 64.4 years \\
\hline
\end{tabular}

${ }^{a}$ The estimated cumulative PGF proportion from the Kaplan-Meier curve at 18 months. ${ }^{\mathrm{b}}$ Glycerol-stored cornea.

surgery, demonstrating the merit of maintained transparency in GISC tissues. This property provides not only a cosmetic benefit to patients but also makes it easier for surgeons to monitor the underlying tube (Figure 1d).

The majority of clinical data on patch materials are derived from retrospective studies with different study populations and length of follow-up. Therefore, comparisons between the various graft material types are difficult. Table 3 shows current PGF rate of GISC in the context of recently published studies. It has been reported that no material used for ADD tube coverage is more prone to erosion than another, including sclera, tutoplast sclera, dura, and tutoplast pericardium. ${ }^{24,27}$ However, in a large comparative case series, Zalta ${ }^{17}$ found that sclera eroded significantly faster than dura after ADD surgery, though the PGF rate suggested that dura and sclera tissues are equally successful in $94 \%$ of ADD surgeries. In the study by Wigton et $a l^{9}$ the glycerol-preserved cornea group presented a significantly lower PGF rate $(1.9 \%$, mean follow-up of 14.5 months) compared with the pericardium group $(8.9 \%$, mean follow-up of
11.0 months). As Table 3 shows, GISC is likely to have a comparable PGF rate and time interval until the occurrence of PGF, comparing to glycerol-preserved cornea, ${ }^{9}$ which might be primarily due to preserved stromal structure in GISC. The study on glycerolpreserved cornea ${ }^{9}$ has much in common with that on optisol-stored fresh cornea, ${ }^{8}$ indicating that the dense, regular collagenous structure of corneal tissue might be particularly suitable for tectonic support of the ocular wall and more resistant to melting than other loosely structured tissues, such as pericardium and sclera. However, both optisol-stored and glycerol-preserved corneas have unfavorable aspects. First, similar to most other donated biological grafts used for ADD coverage, fresh and glycerol-preserved cornea are not completely sterile and carry a potential risk of infectious or neoplastic processes that were not detected during the donation protocol. ${ }^{28-30}$ Zalta $^{17}$ reported that $5 \%$ of cases had positive bacterial cultures at the time of patch graft repair; on the contrary, GISC is processed with gamma irradiation for sterility. As demonstrated in this study, the 
GISC grafts presented no clinical signs of ocular infection after ADD surgery. Second, the use of fresh or glycerolpreserved cornea from the eye bank requires extra timeconsuming intraoperative manipulation (ie, thinning or rehydrating prior to use), while GISC is precut in a split half-moon shape and for immediate use during surgery. Additionally, a glycerol-stored cornea is usually opaque for several weeks after transplantation and an optisolstored fresh cornea has to be used within a short period of time after harvesting. ${ }^{31}$ As is known, GISC has the advantages of transparence and a long-stable shelf life. ${ }^{16}$ Therefore, the rationale for the use of GISC in ADD surgeries is likely that GISC remedies the defects of optisol-stored fresh cornea and glycerol-stored cornea with its unique features.

Tube exposure can open access for bacteria present on the ocular surface to the interior of the eye, which can result in endophthalmitis. There were two cases of presumed endophthalmitis associated with tube exposure related to PGF in the current study, though the microbial cultures were negative. Multiple studies have revealed the link between PGF and endophthalmitis, reinforcing the need to identify potential risk factors for PGF. The pathophysiological mechanisms for PGF are unclear and most likely multifactorial, 7,32,33 including immunemediated inflammation, excessive tension or mechanical rubbing of tissue overlying the tube, poor perfusion, and ischemic damage to the conjunctiva. In our study, we aimed to identify those elements of the patient's preoperative and intraoperative history that could potentially increase the risk of PGF. However, none of the prior predictors demonstrated an impact on the risk of PGF. Byun et $a l^{34}$ reported that previous ocular surgical procedures elevate the risk for PGF. On the other hand, Trubnik et $a l^{35}$ found that it is the concurrent rather than previous ocular surgery that associates with PGF. Current data show that multiple instances of trabeculectomy/ exPress shunt/bleb revision might be a potential risk factor for future PGF but not a significant factor. It is worth noting that PGF is an infrequent occurrence in this cohort, therefore making it difficult to amass enough data for risk analysis.

The primary weakness of this study is its retrospective nature with a relatively short follow-up period (mean15.4 \pm 9.8 (SD) months). Because PGF occurred in only $2.6 \%$ of ADD surgeries over a period of both 12 and 18 months, a prospective comparison of different patch materials would require a long-term study that controlled for examined possible risk factors for PGF. There are other limitations inherent to this retrospective study, including losses to follow-up and incompletely documented data (such as systemic morbidities and detailed description of the grafts). Although the ADD implantation procedure was standardized, slight differences in surgical technique among surgeons were not accounted for. Despite these limitations, the current study represents the most comprehensive examination of PGF after ADD surgery using GISC patch graft.

In conclusion, this is the first multicenter study to our knowledge that extensively evaluates the clinical outcomes of GISC in ADD surgery. GISC is an attractive alternative to traditional graft patch covering ADD implant. It offers sterility advantages, high tensile strength and rigidity, ease of availability, long shelf life, and improved cosmesis owing to its clarity. As GISC becomes increasingly common in ADD surgeries, longterm stability of GISC, including tissue clarity, graft thinning/melting, and any adverse graft-related outcomes, should be evaluated.

\section{Summary}

What was known before

- Gamma-irradiated sterile cornea (GISC) is a relatively new material and the long-term clinical results in coverage of aqueous drainage device (ADD) are limited.

What this study adds

- To better evaluate the safety and effectiveness of the GISC as patch graft in primary ADD surgery, we performed a multicenter retrospective study, paying special attention to the function of GISC as tube coverage to prevent erosion and other GISC-related complications.

\section{Conflict of interest}

Dr Walter J Stark, MD is unpaid Medical Director of Tissue Banks International. Dr Gail Schwartz and Dr Pradeep Ramulu are speakers of Tissue Bank International. All authors have no proprietary or commercial interest in any materials discussed.

\section{Acknowledgements}

We thank Dr Rubamalar Gunatheesan for her efforts on this study. This work was supported in part by Andreas Dreacopolous Eye Research Fund, New York and Raymond Kwok Family Research Fund, USA.

\section{References}

1 Ayyala RS, Zurakowski D, Smith JA, Monshizadeh R, Netland PA, Richards DW et al. A clinical study of the Ahmed glaucoma valve implant in advanced glaucoma. Ophthalmology 1998; 105(10): 1968-1976.

2 Heuer DK, Lloyd MA, Abrams DA, Baerveldt G, Minckler DS, Lee MB et al. Which is better? One or two? A randomized clinical trial of single-plate versus double-plate Molteno 
implantation for glaucomas in aphakia and pseudophakia. Ophthalmology 1992; 99(10): 1512-1519.

3 Freedman J. Scleral patch grafts with Molteno setons. Ophthalmic Surg 1987; 18(7): 532-534.

4 Brandt JD. Patch grafts of dehydrated cadaveric dura mater for tube-shunt glaucoma surgery. Arch Ophthalmol 1993; 111(10): 1436-1439.

5 Rojanapongpun P, Ritch R. Clear corneal graft overlying the seton tube to facilitate laser suture lysis. Am J Ophthalmol 1996; 122(3): 424-425.

6 Singh M, Chew PT, Tan D. Corneal patch graft repair of exposed glaucoma drainage implants. Cornea 2008; 27(10): 1171-1173.

7 Gutiérrez-Díaz E, Montero-Rodríguez M, Mencía-Gutiérrez E, Cabello A, Monescillo J. Long-term persistence of fascia lata patch graft in glaucoma drainage device surgery. Eur J Ophthalmol 2005; 15(3): 412-414.

8 Lama PJ, Fechtner RD. Tube erosion following insertion of a glaucoma drainage device with a pericardial patch graft. Arch Ophthalmol 1999; 117(9): 1243-1244.

9 Wigton E, C Swanner J, Joiner W, Feldman A, McGwin Jr G, Huisingh $\mathrm{C}$ et al. Outcomes of shunt tube coverage with glycerol preserved cornea versus pericardium. J Glaucoma 2014; 23(4): 258-261.

10 Coleman AL, Mondino BJ, Wilson MR, Casey R. Clinical experience with the Ahmed glaucoma valve implant in eyes with prior or concurrent penetrating keratoplasties. Am J Ophthalmol 1997; 123(1): 54-61.

11 Siegner SW, Netland PA, Urban Jr RC, Williams AS, Richards DW, Latina MA et al. Clinical experience with the Baerveldt glaucoma drainage implant. Ophthalmology 1995; 102(9): 1298-1307.

12 Stevenson W, Cheng SF, Emami-Naeini P, Hua J, Paschalis EI, Dana $\mathrm{R}$ et al. Gamma-irradiation reduces the allogenicity of donor corneas. Invest Ophthalmol Vis Sci 2012; 53(11): 7151-7158

13 Utine CA, Tzu JH, Akpek EK. Lamellar keratoplasty using gamma-irradiated corneal lenticules. Am J Ophthalmol 2011; 151(1): 170-174 e171.

14 Daoud YJ, Smith R, Smith T, Akpek EK, Ward DE, Stark WJ The intraoperative impression and postoperative outcomes of gamma-irradiated corneas in corneal and glaucoma patch surgery. Cornea 2011; 30(12): 1387-1391.

15 Akpek EK, Aldave AJ, Aquavella JV. The use of precut, gamma-irradiated corneal lenticules in Boston type 1 keratoprosthesis implantation. Am J Ophthalmol 2012; 154(3): 495-498 e491.

16 Ekici F, Moster MR, Cvintal V, Hu WD, Waisbourd M. Tube shunt coverage with gamma-irradiated cornea allograft (VisionGraft). Clin Ophthalmol 2015; 22(5): 751-755.

17 Zalta $\mathrm{AH}$. Long-term experience of patch graft failure after Ahmed glaucoma valve( $(R)$ surgery using donor dura and sclera allografts. Ophthalmic Surg Lasers Imaging 2012; 43(5): 408-415.

18 Ying Z, Wei LJ. The Kaplan-Meier estimate for dependent failure time observations. J Multivar Anal 1994; 50: 17-29.

19 Raviv T, Greenfield DS, Liebmann JM, Sidoti PA, Ishikawa H, Ritch R. Pericardial patch grafts in glaucoma implant surgery. J Glaucoma 1998; 7(1): 27-32.
20 Nguyen QH, Budenz DL, Parrish RK 2nd. Complications of Baerveldt glaucoma drainage implants. Arch Ophthalmol 1998; 116(5): 571-575.

21 Melamed S, Cahane M, Gutman I, Blumenthal M. Postoperative complications after Molteno implant surgery. Am J Ophthalmol 1991; 111(3): 319-322.

22 Lloyd MA, Baerveldt G, Fellenbaum PS, Sidoti PA, Minckler DS, Martone JF et al. Intermediate-term results of a randomized clinical trial of the 350- versus the 500-mm2 Baerveldt implant. Ophthalmology 1994; 101(8): 1456-1463 discussion 1454-1463.

23 Lim KS, Allan BD, Lloyd AW, Muir A, Khaw PT. Glaucoma drainage devices; past, present, and future. $\mathrm{Br} J$ Ophthalmol 1998; 82(9): 1083-1089.

24 Smith MF, Doyle JW, Ticrney JW. A comparison of glaucoma drainage implant tube coverage. J Glaucoma 2002; 11(2): 143-147.

25 Bailey AJ, Rhodes DN, Cater CW. Irradiation-induced crosslinking of collagen. Radiat Res 22: 606-6211964.

26 Wollensak G, Aurich H, Pham DT, Wirbelauer C. Hydration behavior of porcine cornea crosslinked with riboflavin and ultraviolet A. J Cataract Refract Surg 2007; 33: 516-521.

27 Muir KW, Lim A, Stinnett S, Kuo A, Tseng H, Walsh MM. Risk factors for exposure of glaucoma drainage devices: a retrospective observational study. BMJ Open 2014; 4(5): e004560.

28 Brown P, Rohwer RG, Gajdusek DC. Sodium hydroxide decontamination of Creutzfeldt-Jakob disease virus. N Engl J Med 1984; 310(11): 727.

29 Hamaguchi T, Noguchi-Shinohara M, Nozaki I, Nakamura Y, Sato T, Kitamoto T et al. The risk of iatrogenic Creutzfeldt-Jakob disease through medical and surgical procedures. Neuropathology 2009; 29(5): 625-631.

30 Zou S, Dodd RY, Stramer SL, Strong DM. Probability of viremia with $\mathrm{HBV}, \mathrm{HCV}, \mathrm{HIV}$, and HTLV among tissue donors in the United States. N Engl J Med 2004; 351(8): 751-759.

31 Lindstrom RL, Kaufman HE, Skelnik DL, Laing RA, Lass JH, Musch D et al. Optisol corneal storage medium. Am J Ophthalmol 1992; 114(3): 345-356.

32 Pakravan M, Yazdani S, Shahabi C, Yaseri M. Superior versus inferior Ahmed glaucoma valve implantation. Ophthalmology 2009; 116(2): 208-213.

33 Gedde SJ, Scott IU, Tabandeh H, Luu KK, Budenz DL, Greenfield DS et al. Late endophthalmitis associated with glaucoma drainage implants. Ophthalmology 2001; 108(7): 1323-1327.

34 Byun YS, Lee NY, Park CK. Risk factors of implant exposure outside the conjunctiva after Ahmed glaucoma valve implantation. Jpn J Ophthalmol 2009; 53(2): 114-119.

35 Trubnik V, Zangalli C, Moster MR, Chia T, Ali M, Martinez P et al. Evaluation of risk factors for glaucoma drainage devicerelated erosions: a retrospective case-control study. J Glaucoma 2013; 11(2): 143-147. 